\title{
An Iterative Technique to Solve Fuzzy Delay Integro- Differential Equations
}

T.L. Yookesh ( $\nabla$ renu_yookesh@yahoo.co.in )

Vignan University

T.P. Latchoumi

Vignan University

K.Balamurugan

Vignan University

\section{Research Article}

Keywords: Volterra integro-differential equations, convergence, iterative technique

Posted Date: November 1st, 2021

DOI: https://doi.org/10.21203/rs.3.rs-1019022/v1

License: (1) This work is licensed under a Creative Commons Attribution 4.0 International License.

Read Full License 


\title{
An Iterative Technique to Solve Fuzzy Delay Integro-Differential Equations
}

\author{
${ }^{1 *}$ T.L. Yookesh, ${ }^{2}$ T.P. Latchoumi, ${ }^{3}$ K.Balamurugan \\ ${ }^{1}$ Department of Science and Humanities, VFSTR (Deemed to be University), Guntur, India \\ ${ }^{2}$ Department of Computer Science and Engineering, SRM Institute of Science and Technology, \\ Ramapuram Campus, Chennai, Tamilnadu, India. \\ ${ }^{3}$ Department of Mechanical Engineering, VFSTR (Deemed to be University), Guntur, India \\ E. Mail: ${ }^{1}$ renu_yookesh@yahoo.co.in
}

\begin{abstract}
In this exploration work, we make an assessment on the get together of nonlinear Volterra fuzzy delay integro-differential conditions, with the help of an iterative technique. The outcome is contrasted with the precise outcome with verify the legitimacy and proficiency of the method to deal with nonlinear Volterra fuzzy delay integro-differential condition. To display the suitable highlights of this proposed system, a numerical worldview is represented.
\end{abstract}

\section{Keywords - Volterra integro-differential equations, convergence, iterative technique}

\section{INTRODUCTION}

In mathematics, an integro-differential equation is a condition that includes commonly to integrals and subsidiaries of functions. Delay, which uncovers the past estimations of the capacity, makes modern, pay its enthusiasm on delay integro-differential equations. Researchers and scientists are exploring the point of delay integro-differential equations through their exertion in science applications, for example, heat transfer, dispersion process, neutron dissemination organic breeds concurrent together with expanding and diminishing percentage of producing. Additionally, these sorts of equations start in the regions of material science, science and building applications. A portion of the applications are uneven streamlined features and air versatile wonders, numerous models of populace development, polymer technology, scientific displaying of the dissemination of discrete particles in a violent liquid, populace elements, compartmental frameworks, atomic reactors and numerical demonstrating of genetic marvels. For instance, Volterra [25] was explored the populace development, focusing his investigation on the inherited impacts and a few creators $[5,7,17,18,19,29]$ talked about the integro-differential frameworks.

Recently because of the modern enthusiasm for blur makes their normal results even more unreasonably reasonable. The uses of the assumption of integro-differential conditions of fuzzy delay have been extended [3032]. Fuzzy delay integro-differential equations assume significant job in demonstrating vulnerability of dynamical frameworks. Fuzzy was presented by Zadeh [28]. Fuzzy integro-differential conditions are in practice of eagerness, because of their significance to the assessment of marvels with memory where inexactness is the trademark. $\mathrm{K}$. Balachandran [8] dealt with controllability of nonlinear Volterra integro-differential frameworks and $\mathrm{K}$. 
Balachandran et al. [9] considered the current outcomes for fuzzy delay integro-differential conditions. Toshiyuko Koto [23] discover the dependability of $\Theta$-technique and Runge-Kutta strategies to explain the delay integrodifferential conditions. Discover the answer for the $\mathrm{n}^{\text {th }}$ request fuzzy integro-differential conditions utilizing the iterative strategy is by A. A. Hemeda [14]. H. M. El-Hawary et al. [11] found the numerical answer for delay integro-differential equations with spline and ghostly techniques. Park and Han in [21] examined the truth and peculiarity hypothesis for an answer of fuzzy Volterra fundamental conditions. Allahviranloo et al. in [6] introduced another technique for tackling fuzzy integro-differential equation under complete differentiability. After that various experts discussed the procedure for courses of action of these sorts of conditions, in view of different quick to fuzzy numbers, there are a couple of research systems to look at fuzzy conditions. [9,14,22, 24].

This paper proposed a strategy dependent on iterative system called an Adomian decomposition technique to illuminate the Volterra delay integro-differential equations with fuzzy. Center our exploration is to discover how this proposed strategy chips away at Volterra delay integro-differential conditions under fuzzy foundation. These procedures join rapidly towards the precise plan and this technique does exclude any discretization of factors and consequently, it is exempted from changing off slip-ups. We embody a numerical manual to explain the proposed system.

\section{PRELIMERIES}

\section{A. Membership Function}

Give $\delta$ a chance to be a nonempty set. A fuzzy set $\overline{\mathrm{A}}$ in $\delta$ is characterized by its membership function $\mu_{\overline{\mathrm{A}}}: \delta \rightarrow[0,1]$ and $\mu_{\overline{\mathrm{A}}(\mathrm{x})}$ is explained as the degree of membership of element $x$ in fuzzy set $\overline{\mathrm{A}}$ for each $x \in \delta$. It is clear that $\bar{A}$ is determined by the set of tuples $\overline{\mathrm{A}}=\left\{\left(x, \mu_{\overline{\mathrm{A}(\mathrm{x})}}\right) \mid x \in \delta\right\}$.

\section{B. Triangular Fuzzy Number}

Let the triangular fuzzy number of fuzzy set $\overline{\mathrm{A}}$ with mid as $c$, left $\operatorname{span} m>0$ and right $\operatorname{span} n>0$, then

$$
\mu(x)=\left\{\begin{array}{ccc}
1-\frac{c-x}{m}, & \text { if } & c-m<x<c \\
1-\frac{x-c}{n}, & \text { if } & c<x<c+n \\
0 & \text { or else }
\end{array}\right.
$$




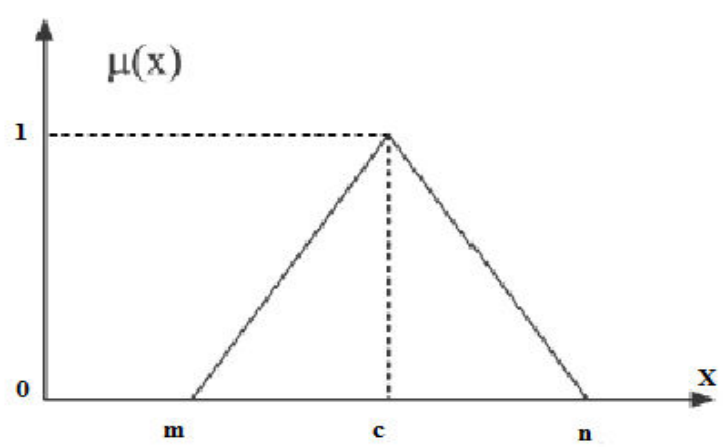

Fig. 1. Triangular Fuzzy Number

\section{NONLINEAR VOLTERRA FUZZY DELAY INTEGRO-DIFFERENTIAL EQUATIONS}

The delay Volterra integro-differential equation of the second kind is stated by,

$$
\psi^{\prime}(q)=p(q)+\int_{a}^{q} k(q, v) P(\psi(v), \psi(v-\tau)) d v
$$

with initial condition $\psi(a)=c_{1}$, where ' $a$ ' is a real constant, $k(q, v)$ and $p(q)$ are real valued functions. The functions $P(\psi(v))$ and $P(\psi(v-\tau))$ are nonlinear function and nonlinear delay function of $\psi(v)$ respectively. If $p(q)$ is a crisp function then the solutions of equation (3.1) are also crisp. However, if $p(q)$ is a fuzzy function this equation may posses only fuzzy solutions. Let us introduce $\alpha$-level set of $\psi, p$ and $P$ to the above equation and we obtain,

$$
\begin{aligned}
& \psi(q, \alpha)=[\underline{\psi}(q, \alpha), \bar{\psi}(q, \alpha)] \\
& \psi(q, \alpha)=\left[\underline{\psi}^{\prime}(q, \alpha), \overline{\psi^{\prime}}(q, \alpha)\right] \\
& p(q, \alpha)=[\underline{p}(q, \alpha), \bar{p}(q, \alpha)] \\
& P(\underline{\psi}(v, \alpha), \bar{\psi}(v, \alpha), \underline{\psi}(v-\tau, \alpha), \bar{\psi}(v-\tau, \alpha))=[G(\underline{\psi}(v, \alpha), \bar{\psi}(v, \alpha), \underline{\psi}(v-\tau, \alpha), \bar{\psi}(v-\tau, \alpha)), \\
& H(\underline{\psi}(v, \alpha), \bar{\psi}(v, \alpha), \underline{\psi}(v-\tau, \alpha), \bar{\psi}(v-\tau, \alpha))]
\end{aligned}
$$

where $0 \leq \alpha \leq 1$ and $q \in[a, b]$

Moreover all the derivatives with respective to $q$, are fuzzy functions. Now, the equation of (3.1) can be written as follows

$$
\left\{\begin{array}{l}
\underline{\psi}^{\prime}(q)=\underline{p}(q)+\int_{a}^{q} k(q, v) \underline{P}(\psi(v), \psi(v-\tau)) d v \\
\bar{\psi}^{\prime}(q)=\bar{p}(q)+\int_{a}^{q} k(q, v) \bar{P}(\psi(v), \psi(v-\tau)) d v
\end{array}\right.
$$

for $0 \leq \alpha \leq 1$. Suppose $k(q, v)$ be continuous in $a \leq v \leq b$ and for fix $q$, the sign of $k(q, v)$ does not change over $[a, q]$, therefore we have 


$$
\begin{aligned}
& \underline{\psi}^{\prime}(q)=\underline{p}(q)+\int_{a}^{q} k(q, v) G(\underline{\psi}(v, \alpha), \bar{\psi}(v, \alpha), \underline{\psi}(v-\tau, \alpha), \bar{\psi}(v-\tau, \alpha)) d v \\
& \bar{\psi}^{\prime}(q)=\bar{p}(q)+\int_{a}^{q} k(q, v) H(\underline{\psi}(v, \alpha), \bar{\psi}(v, \alpha), \underline{\psi}(v-\tau, \alpha), \bar{\psi}(v-\tau, \alpha)) d v
\end{aligned}
$$

with subject to initial condition $[\underline{\psi}(v, \alpha), \bar{\psi}(v, \alpha)]=\left[\underline{b_{1}}(\alpha), \overline{b_{1}}(\alpha)\right]$ for each $0 \leq \alpha \leq 1$ and $v \in[a, b]$. We can see that equation (3.4) are arangement of Volterra fuzzy delay integro-differential equations in the crisp case for each $0 \leq \alpha \leq 1$.

\section{DESCRIPTION OF THE METHOD}

Here, we clarify the principle calculation of Adomian decomposition technique [2, 3] that applies to a Volterra fuzzy delay integro-differential equations $[1,27]$ of the structure

$$
\begin{aligned}
& \underline{\psi^{\prime}}(q, \alpha)=\underline{f}(q, \alpha)+\int_{a}^{q} k(q, v) G[\underline{\psi}(v, \alpha), \bar{\psi}(v, \alpha), \underline{\psi}(v-\tau, \alpha), \bar{\psi}(v-\tau, \alpha)] d v \\
& \bar{\psi}^{\prime}(q, \alpha)=\bar{f}(q, \alpha)+\int_{a}^{q} k(q, v) H[\underline{\psi}(v, \alpha), \bar{\psi}(v, \alpha), \underline{\psi}(v-\tau, \alpha), \bar{\psi}(v-\tau, \alpha)] d v
\end{aligned}
$$

The Volterra fuzzy delay integro-differential equations can be written as follows

$$
\begin{gathered}
\underline{\psi}^{\prime}(q, \alpha)=\underline{f}(q, \alpha)+\int_{a}^{q} k(q, v) G[\underline{\psi}(v, \alpha), \bar{\psi}(v, \alpha), \underline{\psi}(v-\tau, \alpha), \bar{\psi}(v-\tau, \alpha)] d v \\
\bar{L}^{\prime}(q, \alpha)=\bar{f}(q, \alpha)+\int_{a}^{q} k(q, v) H[\underline{\psi}(v, \alpha), \bar{\psi}(v, \alpha), \underline{\psi}(v-\tau, \alpha), \bar{\psi}(v-\tau, \alpha)] d v
\end{gathered}
$$

where $L$ be the derivative with respect to $q$ and $G[\underline{\psi}(v, \alpha), \bar{\psi}(v, \alpha), \underline{\psi}(v-\tau, \alpha), \bar{\psi}(v-\tau, \alpha)]$ and $H[\underline{\psi}(v, \alpha), \bar{\psi}(v, a), \underline{\psi}(v-\tau, \alpha), \bar{\psi}(v-\tau, a)]$ are nonlinear terms. Now handle the inverse operator $L^{-1}$ to two hands of (4.2), then we have the form

$$
\begin{aligned}
& \underline{\psi}^{\prime}(q, \alpha)=\underline{g}(q, \alpha)+L^{-1}\left(\int_{a}^{q} k(q, v) G[\underline{\psi}(v, \alpha), \bar{\psi}(v, \alpha), \underline{\psi}(v-\tau, \alpha), \bar{\psi}(v-\tau, \alpha)] d v\right) \\
& \bar{\psi}^{\prime}(q, \alpha)=\bar{g}(q, \alpha)+L^{-1}\left(\int_{a}^{q} k(q, v) H[\underline{\psi}(v, \alpha), \bar{\psi}(v, \alpha), \underline{\psi}(v-\tau, \alpha), \bar{\psi}(v-\tau, \alpha)] d v\right)
\end{aligned}
$$

where the functions $L^{-1} \underline{f}(q, \alpha)=\underline{g}(q, \alpha)$ and $L^{-1} \bar{f}(q, \alpha)=\bar{g}(q, \alpha)$. The ADM expect an unbounded arrangement answer for the obscure capacities $[\underline{\psi}(q, \alpha), \bar{\psi}(q, a)]$, given by 


$$
\begin{aligned}
& \underline{\psi}(v, \alpha)=\sum_{i=0}^{\infty} \underline{\psi}_{i}(q, \alpha) \text { and } \\
& \bar{\psi}(v, a)=\sum_{i=0}^{\infty} \bar{\psi}_{i}(q, \alpha)
\end{aligned}
$$

Then onlinear operators $G[\underline{\psi}(v, \alpha), \bar{\psi}(v, \alpha), \underline{\psi}(v-\tau, \alpha), \bar{\psi}(v-\tau, \alpha)], H[\underline{\psi}(v, \alpha), \bar{\psi}(v, \alpha), \underline{\psi}(v-\tau, \alpha), \bar{\psi}(v-\tau, \alpha)]$ into an unending arrangement of polynomials given by

$$
\begin{aligned}
& G[\underline{\psi}(v, \alpha), \bar{\psi}(v, a)]=\sum_{i=0}^{\infty} \underline{A}_{n} ; G[\underline{\psi}(v-\tau, \alpha), \bar{\psi}(v-\tau, a)]=\sum_{i=0}^{\infty} \bar{A}_{n} \text { and } \\
& H[\underline{\psi}(v, \alpha), \bar{\psi}(v, a)]=\sum_{i=0}^{\infty} \underline{B}_{n} ; H[\underline{\psi}(v-\tau, \alpha), \bar{\psi}(v-\tau, a)]=\sum_{i=0}^{\infty} \bar{B}_{n}
\end{aligned}
$$

Where $A_{n}=\left[A_{n} ; \overline{A_{n}}\right], B_{n}=\left[\underline{B_{n}}, \overline{B_{n}}\right], n \geq 0$ are the Adomian polynomial characterize by,

$$
\begin{aligned}
& \underline{A}_{n}=\frac{1}{n !}\left[\frac{d^{n}}{d \lambda^{n}} G\left(\sum_{i=0}^{n} \lambda^{i} \underline{\psi}_{i}, \sum_{i=0}^{n} \lambda^{i} \bar{\psi}_{i}\right)\right]_{\lambda=0} \\
& \bar{A}_{n}=\frac{1}{n !}\left[\frac{d^{n}}{d \lambda^{n}} G\left(\sum_{i=0}^{n} \lambda^{i} \underline{\psi}_{i}, \sum_{i=0}^{n} \lambda^{i} \bar{\psi}_{i}\right)\right]_{\lambda=0} \\
& \underline{B}_{n}=\frac{1}{n !}\left[\frac{d^{n}}{d \lambda^{n}} H\left(\sum_{i=0}^{n} \lambda^{i} \underline{\psi}_{i}, \sum_{i=0}^{n} \lambda^{i} \overline{\psi_{i}}\right)\right]_{\lambda=0} \\
& \bar{B}_{n}=\frac{1}{n !}\left[\frac{d^{n}}{d \lambda^{n}} H\left(\sum_{i=0}^{n} \lambda^{i} \underline{\psi}_{i}, \sum_{i=0}^{n} \lambda^{i} \overline{\psi_{i}}\right)\right]_{\lambda=0}
\end{aligned}
$$

On subsituting (4.4) and (4.5) into (4.3), we get

$$
\begin{aligned}
& \underline{\psi}_{0}=\underline{g}(q, \alpha), \\
& \underline{\psi}_{n+1}=L^{-1} \int_{a}^{q} k(q, v) \underline{A}_{n} d v, n \geq 0
\end{aligned}
$$

and

$$
\begin{aligned}
& \bar{\psi}_{0}=\bar{g}(q, \alpha) \\
& \bar{\psi}_{n+1}=L^{-1} \int_{a}^{q} k(q, v) \bar{B} n d v, n \geq 0
\end{aligned}
$$


We approximate $\psi=[\underline{\psi}(q, \alpha), \bar{\psi}(q, \alpha)]$ by $\underline{\varphi}=\sum_{i=0}^{n-1} \underline{\psi}_{i}(q, \alpha) ; \bar{\varphi}=\sum_{i=0}^{n-1} \bar{\psi}_{i}(q, \alpha)$, where $\lim _{n \rightarrow \infty} \underline{\varphi}=\underline{\psi_{i}}(q, \alpha)$ and $\lim _{n \rightarrow \infty} \bar{\varphi}=\overline{\psi_{i}}(q, \alpha)$

\section{UNIQUENESS AND CONVERGENCE OF THE METHOD}

\section{A. Uniqueness theorem}

If $\underline{\psi}(q)=\underline{p}(q)+\int_{0}^{q-\tau} \underline{P}(\psi(q)) d v ; \bar{\psi}(q)=\bar{p}(q)+\int_{0}^{q-\tau} \bar{P}(\psi(q)) d v$ has a unique solutions whenever $0<\alpha<1$, where $\alpha=L M T$.

Proof: Let $\underline{\psi}, \underline{\psi^{*}} ; \bar{\psi}, \overline{\psi^{*}}$ are the two different solutions to $\underline{\psi}(u) ; \bar{\psi}(u)$, then

$$
\begin{aligned}
\left|\underline{\psi}-\underline{\psi^{*}}\right| & =\left|\int_{0}^{q-\tau} k(u, v)\left[\underline{F}(\psi)-\underline{F}^{*}(\psi)\right] d v\right| ; \\
\left|\bar{\psi}-\overline{\psi^{*}}\right| & =\left|\int_{0}^{q-\tau} k(u, v)\left[\bar{F}(\psi)-\bar{F}^{*}(\psi)\right] d v\right| \\
& \leq \int_{0}^{q-\tau}|k(u, v)|\left|\left[\underline{F}(\psi)-\underline{F}^{*}(\psi)\right]\right| d v ; \\
& \int_{0}^{q-\tau}|k(u, v)|\left|\left[\bar{F}(\psi)-\bar{F}^{*}(\psi)\right]\right| d v \\
& \leq \alpha M\left|\underline{\psi}-\underline{\psi}^{*}\right| \int_{0}^{q-\tau} d v ; L M\left|\bar{\psi}-\bar{\psi}^{*}\right| \int_{0}^{q-\tau} d v \\
& \leq \alpha\left|\underline{\psi}-\underline{\psi^{*}} \| ; \alpha\right| \bar{\psi}-\bar{\psi}^{*} \mid
\end{aligned}
$$

from which we get $(1-\alpha)\left|\underline{\psi}-\underline{\psi^{*}}\right| \leq 0$ and $(1-\alpha)\left|\bar{\psi}-\overline{\psi^{*}}\right| \leq 0$ implies $\underline{\psi}=\underline{\psi^{*}} ; \bar{\psi}=\overline{\psi^{*}}$.

\section{B. Convergence theorem}

The arrangement $\bar{\psi}(w, \alpha)=\sum_{i=0}^{\infty} \bar{\psi}_{i}(w, \alpha)$ of the problem (4.2) using ADM convergence when $0<\alpha<1,\left|\psi_{1}(w, \alpha)\right|<\infty$.

Proof: Confine the series of partial sums $\overline{\varphi_{n}}$, let $\bar{\varphi}_{n}$ and $\overline{\varphi_{m}}$ be arbitrary partial sums with $n \geq m$. We have to show that $\overline{\varphi_{n}}$ is a Cauchy alignment in this Banach space. 
Now,

$$
\begin{aligned}
\left\|\overline{\varphi_{n}}-\overline{\varphi_{m}}\right\| & =\max _{\forall x \in[a, b]}\left|\overline{\varphi_{n}}-\overline{\varphi_{m}}\right| \\
& =\max _{\forall x \in[a, b]}\left|\sum_{i=m+1}^{n} \overline{\psi_{i}}(w, \alpha)\right| \\
& =\max _{\forall x \in[a, b]}\left|\sum_{i=m+1}^{n}\left(\int_{a}^{r} k(w, v) \overline{\overline{A_{i}}} d v\right)\right| \\
& =\max _{\forall x \in[a, b]}\left|\int_{a}^{r} k(w, v)\left(\sum_{i=m+1}^{n} \overline{\overline{A_{i}}}\right) d v\right|
\end{aligned}
$$

From [10], we know

$$
\sum_{i=m}^{n-1} \overline{\overline{A_{i}}}=G\left(\underline{\varphi}_{n-1}, \bar{\varphi}_{n-1}\right)-G\left(\underline{\varphi}_{m-1}, \bar{\varphi}_{m-1}\right) .
$$

So,

$$
\begin{aligned}
\left\|\overline{\varphi_{n}}-\overline{\varphi_{m}}\right\| & =\left.\max _{\forall x \in[a, b]}\right|_{a} ^{r} k(w, v)\left(G\left(\underline{\varphi}_{n-1}, \bar{\varphi}_{n-1}\right)-G\left(\underline{\varphi}_{m-1}, \bar{\varphi}_{m-1}\right)\right) d v \mid \\
& \leq \int_{a}^{r}|k(w, v)|\left|G\left(\underline{\varphi}_{n-1}, \bar{\varphi}_{n-1}\right)-G\left(\underline{\varphi}_{m-1}, \bar{\varphi}_{m-1}\right)\right| d v \\
& \leq j\left\|\overline{\varphi_{n}}-\overline{\varphi_{m}}\right\|
\end{aligned}
$$

Take $n=m+1$, we have

$$
\begin{aligned}
&\left\|\overline{\varphi_{n}}-\overline{\varphi_{m}}\right\| \leq j\left\|\bar{\varphi}_{m}-\bar{\varphi}_{m-1}\right\| \\
& \leq j\left\|\bar{\varphi}_{m-1}-\bar{\varphi}_{m-2}\right\| \\
& \vdots \\
& \leq j\left\|\bar{\varphi}_{1}-\bar{\varphi}_{0}\right\|
\end{aligned}
$$

and 


$$
\begin{aligned}
\left\|\overline{\varphi_{n}}-\overline{\varphi_{m}}\right\| & \leq\left\|\bar{\varphi}_{m+1}-\bar{\varphi}_{m}\right\|+\left\|\bar{\varphi}_{m+2}-\bar{\varphi}_{m+1}\right\|+\cdots+\left\|\bar{\varphi}_{n}-\bar{\varphi}_{n-1}\right\| \\
& \leq\left|j^{m}+j^{m+1}+\cdots+j^{n-1}\right|\left\|\bar{\varphi}_{1}-\bar{\varphi}_{0}\right\| \\
& \leq\left|j^{1}+j^{2}+\cdots+j^{n-m-1}\right|\left\|\bar{\varphi}_{1}-\bar{\varphi}_{0}\right\| \\
& \leq j^{m}\left[\frac{1-j^{n-m}}{1-j}\right]\left\|\bar{\psi}_{1}(w, \alpha)\right\|
\end{aligned}
$$

Since $0<j<1$, we have $1-j^{n-m}<1$ then $\left\|\overline{\varphi_{n}}-\overline{\varphi_{m}}\right\| \leq \max _{\forall x \in[a, b]}\left|\overline{\psi_{1}}(w, \alpha)\right|$. But $\left|\overline{\psi_{1}}(w, \alpha)\right|<\infty$. So as $m \rightarrow \infty,\left\|\overline{\varphi_{n}}-\overline{\varphi_{m}}\right\| \rightarrow 0$. We finalished that $\overline{\varphi_{n}}$ is Cauchy allignment, so $\bar{\psi}(w, \alpha)=\lim _{n \rightarrow \infty} \bar{\psi}(w, \alpha)$.

Likewise, we admit $\underline{\varphi}_{n}$ is a Cauchy allignment. Then, we address by $\underline{\psi}(w, \alpha)=\lim _{n \rightarrow \infty} \underline{\psi}(w, \alpha)$. Therefore, $\overline{\bar{\psi}}(w, \alpha)=\lim _{n \rightarrow \infty} \overline{\bar{\psi}}(w, \alpha)$. So the series is converges.

\section{NUMERICAL EXAMPLE}

Here, we show the iterative effectiveness of ADM nonlinear Volterra fuzzy delay integro-differential equations with a analytical precedent is outlined. Give us a chance to study the nonlinear Volterra fuzzy delay integro-differential equation has the accompanying structure

$$
\begin{aligned}
& \underline{\psi^{\prime}}(r, \alpha)=\frac{\alpha^{2}}{2}+\alpha e^{r}-\frac{\alpha^{2}}{2} e^{2 r}+\int_{0}^{r} \underline{\psi^{2}}\left(\frac{t}{2}, \alpha\right) d t \\
& \overline{\psi^{\prime}}(r, \alpha)=2 e^{r}-\alpha e^{r}+2-2 \alpha+\frac{\alpha^{2}}{2}+2 \alpha e^{2 r}+\int_{0}^{r \overline{\psi^{2}}}\left(\frac{t}{2}, \alpha\right) d t
\end{aligned}
$$

with initial conditions $\quad \underline{\psi}(0 ; \alpha)=\alpha$ and $\bar{\psi}(0 ; \alpha)=2-\alpha . \quad$ Has $\quad$ the exact solution, $\underline{\zeta}(r ; \alpha)=\alpha e^{r}, \bar{\zeta}(r ; \alpha)=(2-\alpha) e^{r}$. On using the series (4.4) and (4.5) into the right and left span of Eq.(6.1) respectively gives,

$$
\begin{aligned}
& \underline{\psi^{\prime}}(r, \alpha)= \alpha+\frac{\alpha^{2}}{2} r+\alpha e^{r}-\frac{\alpha^{2}}{4} e^{2 r}+\int_{0}^{r} A_{n} d t \\
& \overline{\psi^{\prime}}(r, \alpha)=2-\alpha+2 e^{r}-\alpha e^{r}+2 r-2 \alpha r \\
&+\frac{\alpha^{2}}{2} r-e^{2 r}+\alpha e^{2 r}-\frac{\alpha^{2}}{4} e^{2 r}+\int_{0}^{r} \overline{A_{n}} d t
\end{aligned}
$$

On solving the Eq. (6.2) with the considered iterative method, will give the inexact solutions and the outcomes are organized in Table 1 . It shows the numerical results at $r=0.5$ which is very close to the exact solution. 
Table 1. Observation between Exact and Approximate values

\begin{tabular}{|c|c|c|c|c|c|c|}
\hline & \multicolumn{2}{|c|}{ Exact Solution } & \multicolumn{2}{c|}{ Approximate Solution } & \multicolumn{2}{c|}{ Error Estimation } \\
\hline$\alpha$ & $\underline{\zeta}(r)$ & $\bar{\zeta}(r)$ & $\underline{\psi}(r)$ & $\bar{\psi}(r)$ & $\underline{E}(r)$ & $\bar{E}(r)$ \\
\hline 0 & 0 & 3.29744 & 0 & 3.27581 & 0 & 0.02158 \\
\hline 0.2 & 0.32974 & 2.96770 & 0.32851 & 2.92819 & 0.00123 & 0.03946 \\
\hline 0.4 & 0.65949 & 2.63795 & 0.65455 & 2.59895 & 0.00493 & 0.03896 \\
\hline 0.6 & 0.98923 & 2.30821 & 0.97811 & 2.28761 & 0.01111 & 0.02057 \\
\hline 0.8 & 1.31898 & 1.97847 & 1.29919 & 1.94392 & 0.01976 & 0.03452 \\
\hline 1.0 & 1.64872 & 1.64872 & 1.61779 & 1.61779 & 0.03090 & 0.03090 \\
\hline
\end{tabular}

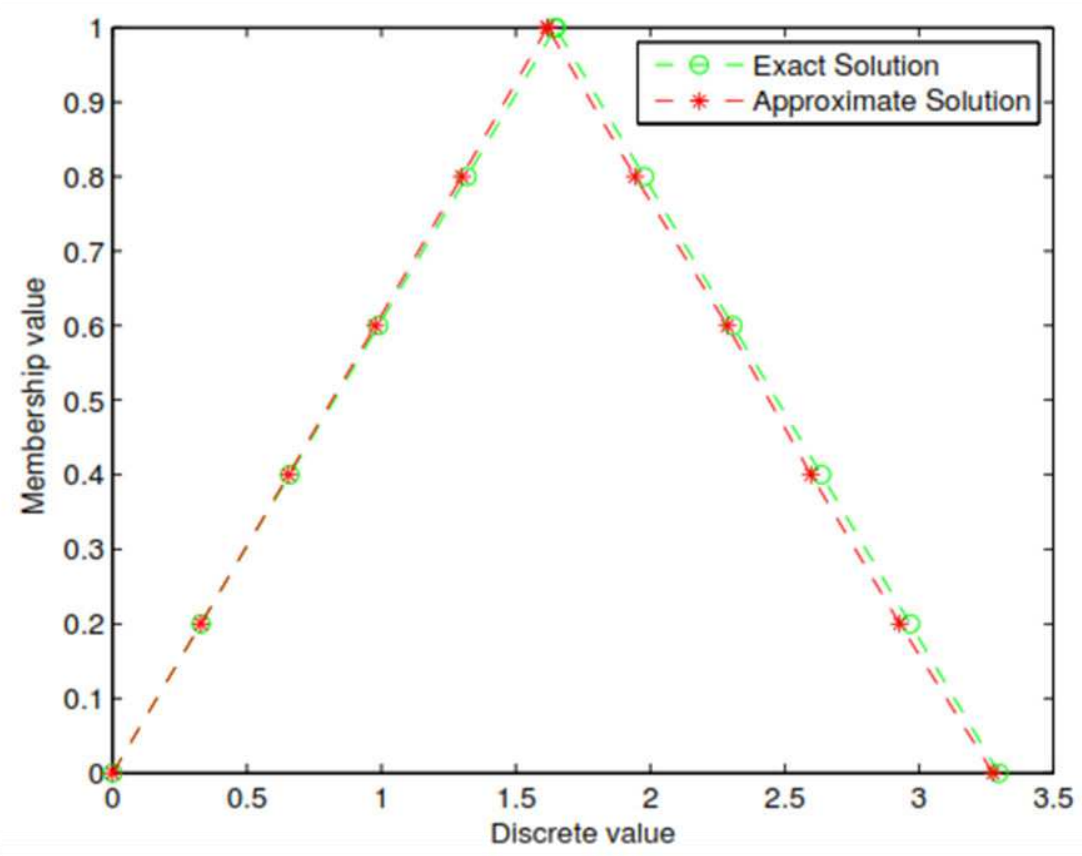

Fig.2. Graphical representation of solutions at $\mathrm{x}=0.5$

Estimated arrangements of nonlinear Volterra Fuzzy delay integro-differential conditions are discovered utilizing ADM from the investigation. Fig 2, demonstrates the graphical portrayal of similarity among estimated and precise arrangements. It built up the union quality of the proposed technique. 


\section{CONCLUSION}

In this paper, a surmised arrangement of nonlinear Volterra Fuzzy postponement integro-differential conditions is discovered utilizing ADM is indented from the examination. For delineation, a precedent is chosen to demonstrate the computational exactness. It might be inferred that the Adomian disintegration strategy is exceptionally incredible and proficient in finding surmised answers for wide classes of issues. The arrangements acquired by the present strategy are consistently focalized. As appeared in Tables 1 . The present investigation has affirmed that the Adomian disintegration strategy offers huge points of interest as far as its direct pertinence, its computational viability, and its exactness to explain the unequivocally straight conditions.

\section{REFERENCES}

[1] Abdul-Majid Wazwaz, Linear and nonlinear integral equations- methods and applications, Higher education press: Springer, Beijing, 2011.

[2] G. Adomian, A review of the decomposition method in applied mathematics, Journal of Mathematical Analysis and Applications, 135 (1988), 501-544.

[3] G. Adomian and R. Rech, Generalization of Adomian polynomials to functions of several variables,Computers \& Mathematics with Applications, 24 (1992), 11-24.

[4] G. Adomian, Solving frontier problems of physics: The decomposition method, Kluwer Academic Publishers, Boston, 1994.

[5] S. Alao, F.S. Akinboro, F.O. Akinpelu and R.A. Oderinu, Numerical solution of integro-differential equation using Adomian decomposition and variational iteration methods, IOSR Journal of Mathematics, 10 (2014), 18-22.

[6] T. Allahviranloo, S. Abbasbandy, O. Sedaghatfar and P. Darabi, A new method for solving fuzzy integro-differential equation under generalized differeniability, Neural Computing and Applications, 21 (2012), 191-196.

[7] Azzeddine Belloura and Mahmoud Bousselsalb, Numerical solution of delay integro-differential equations by using Taylor collocation method, Mathematical Methods in the Applied Sciences, 37 (2014), 1491-1506.

[8] K. Balachandran, Controllability of nonlinear Volterra integro-differential systems, Kybernatika, 25 (1989), 505-508.

[9] K.Balachandran and K. Kanagarajan, Existence of solutions of fuzzy delay integro-differential equations with nonlocal conditions, The Korean Society for Industrial and Applied Mathematics, 9 (2005), 333-343. 
[10] I. L. El-Kalaa, Convergence of the Adomain method applied to a class of nonlinear integral equations, Applied Mathematics Letters, 21 (2008), 372-376.

[11] H.M. El-Hawary, K.A. El-Shami, Numerical solution of Volterra delay-integrodifferential equations via spline/spectral methods, International Journal of Differential Equations and Application, 12 (2013), 149-157.

[12] M. Ghanbri, Numerical solution of fuzzy linear Volterra integral equations of the second kind by Homotopy analysis method, International Journal of Mathematics, 2 (2010), 73-87.

[13] Hermann Brunner, The numerical treatment of Volterra integro-differential equations with unbounded delay, Computational and Applied Mathematics, 28 (1989), 5-23.

[14] A. A. Hemeda, Formulation and solution of n-th-Order derivative fuzzy integro-differential equation using new iterative method with a reliable algorithm, Journal of Applied Mathematics, 2012 (2012), 1-17.

[15] O. Kaleva, The Cauchy problem for fuzzy differential equations, Fuzzy Sets and Systems, 35 (1990), 389-396.

[16] O. Kaleva, A note on fuzzy differential equations, Nonlinear Analysis, 64 (2006), 895-900.

[17] Kamel Al-Khaled and Fathi Alllan, Decomposition method for solving nonlinear integrodifferential equations, Journal of Applied Mathematics and Computing, 19 (2005), 415-425.

[18] A. Mohsen and M. El-Gamel, On the numerical solution of linear and nonlinear volterra integral and integro-differential equations, Applied Mathematics and Computation, 217 (2010), 3330-3337.

[19] Mohammad Bagher Moghimi and Abdollah Borhanifar, Solving a class of nonlinear delay integro-differential equations by using differential equations transformation method, Applied and Computational Mathematics, 5 (2016), 142-149.

[20] S. K. Ntouyas, Global existence results for certain second order delay integrodifferential equations with nonlocal conditions, Dynamic Systems and Applications, 7 (1998), 415-425.

[21] J. Y. Park and H. K. Han, Existence and uniqueness theorem for a solution of fuzzy Volterra integral equations, Fuzzy Sets and Systems, 105 (1999), 481-488.

[22] S. Parthiban and P. Ganivaradhan, Statistical hypothesis test in three factor ANOVA model under fuzzy environments using trapezoidal fuzzy numbers, Bulletin of Mathematical Sciences and Applications, 14 (2016), 23-44.

[23] Toshiyuki Koto, Stability of Runge-Kutta methods for delay integrodifferential equations, Computational and Applied Mathematics, 145 (2002), 483-492.

[24] S. Vinoth and K. Arjunan, A Study on Interval Valued Intuitionistic Fuzzy Generalized Semipreclosed Sets, International Journal of Fuzzy Mathematics and Sytems, 5 (2015), 47-55. 
[25] V. Volterra, Theory of functional and integral and integro-differential equations, Dover, New york, 1959.

[26] A. M. Wazwaz, Linear and Nonlinear Integral Equations: Methods and Applications, Springer Berlin Heidelberg, Newyork, 2011.

[27] T.L.Yookesh, M.Chithambarathanu, E.Boopathi Kumar and P.Sudam Sekhar, Efficiency of iterative filtering method for solving Volterra fuzzy integral equations with a delay and material investigation, Materials today: Proceedings, 47 (2021), 6101-6104.

[28] L. A. Zadeh, Fuzzy sets, Information and Control, 8 (1965), 338-353.

[29] M. Zarebnia and M. G. Ali Abadi, Numerical solution of system of nonlinear second-order integro-differential equations, Computers \& Mathematics with Applications, 60 (2010), 591-601.

[30] S. Noeiaghdam and M. A. F. Araghi, Application of the CESTAC Method to Find the Optimal Iteration of the Homotopy Analysis Method for Solving Fuzzy Integral Equations. In International Online Conference on Intelligent Decision Science, 2020, 623-637.

[31] H.S. Hadeed., A.S. Hamad and A.A. Hamoud, Numerical Iterative Methods for Solving Nonlinear Volterra-Fredholm Integral Equations. Advances in Dynamical Systems and Applications (ADSA), 16(2021), 535-545.

[32] M.B. Issa and A. Hamoud, Some approximate methods for solving system of nonlinear integral equations. Technology Reports of Kansai University, 62(2020), 388-398. 